\title{
Resumption Lag at Interruptible Timing might not be short in Actual Environment
}

\author{
Takahiro Tanaka \\ Kazuaki Aoki \\ Tokyo University of Agriculture and Tokyo University of Agriculture and \\ Technology \\ Technology \\ Naka-machi 2-24-16, Koganei-shi, Naka-machi 2-24-16, Koganei-shi, \\ Tokyo, Japan \\ Tokyo, Japan \\ takat@cc.tuat.ac.jp \\ kazuak@cc.tuat.ac.jp \\ Niels A. Taatgen \\ University of Groningen \\ Kinya Fujita \\ Nijenborgh 9, 9747 AG Groningen, Technology \\ Netherlands \\ Naka-machi 2-24-16, Koganei-shi, \\ n.a.taatgen@ai.rug.n! \\ Tokyo, Japan \\ kfujita@cc.tuat.ac.jp
}

Permission to make digital or hard copies of part or all of this work for personal or classroom use is granted without fee provided that copies are not made or distributed for profit or commercial advantage and that copies bear this notice and the full citation on the first page. Copyrights for third-party components of this work must be honored. For all other uses, contact the Owner/Author.

Copyright is held by the owner/author(s).

CHI 2014, Apr 26 - May 01 2014, Toronto, ON, Canada ACM 978-1-4503-2474-8/14/04.

http://dx.doi.org/10.1145/2559206.2581207

\begin{abstract}
Experiments performed in controlled environments have revealed that interruptions occurring at application switching (AS), which correspond to task breakpoints in the computer field, are more subjectively acceptable and require shorter resumption lags (RL), which indicate the cognitive cost. Therefore, in this study, we investigate RLs in uncontrolled office environment. The interruptions at more acceptable ASs were expected to show shorter RLs, because the cognitive costs of the interruption are less. However, contrary to the expected result, the RLs were longer. This implies that factors other than cognitive cost may affect interruptibility in a realistic environment.
\end{abstract}

\section{Author Keywords}

Interruptibility; cognitive cost; task breakpoint; application switching.

\section{ACM Classification Keywords}

H.5.2 [User Interface]: Theory and methods.

\section{Introduction}

With the increasing popularity of the Internet and information systems, online communication and data access have become more important to our daily lives. 
Meanwhile, the possibility of users getting interrupted while using information systems has also been increasing. However, the timing or frequency of interruptions by information systems has not been controlled, and frequent interruptions that do not reflect the user status can fragment the user's work time and reduce intellectual productivity [6].

Studies on humans multitasking suggest that switching between tasks requires the suspension and resumption of memory processes associated with the suspended task, and thus causes a "resumption lag (RL)" [1,9]. It has also been reported that the RL increases when the amount of information required for switching tasks becomes large. Thus, previous studies have used the $\mathrm{RL}$ to evaluate the cognitive cost caused by task switching and interruptions $[6,8]$. On the other hand, the amount of information passing via the user's intentional task breakpoints decreases because the preceding task has already been suspended. It has been shown experimentally that the $\mathrm{RL}$ for interruptions, which are made at task breakpoints, are significantly shorter than those for interruptions during continuous work [10]. Thus, the breakpoints have the possibility to cause timing interruptions with less cognitive impact.

The effects of task breakpoints have been investigated based on prior task-structure analyses [4]. Other studies focused on application switching (AS) as the task breakpoint during PC work $[2,3]$. Because AS is a type of self-interruption [5], the suspension and resumption of working memories can be smoothly achieved. Therefore, the cognitive cost of interruptions at AS is expected to be less compared with interruptions occurring when not application switching (NAS). Moreover, the interruptions occurring at AS are more acceptable [11].

The previous studies on the cognitive costs were performed under controlled conditions in terms of tasks and instructions. However, the conditions of actual tasks and subjects may vary in real office environments. In this study, to validate the phenomenon suggested by the previous studies, we analyzed the cognitive costs of interruptions based on the RL in an uncontrolled environment. The results supported the expected relationship between task phase and cognitive cost. However, the results also suggested that the RLs for acceptable interruptions are longer than those for unacceptable interruptions, which is contrary to the expected result.

\section{Experiment: Collecting Operation Records}

In this study, we developed and installed a softwarebased experimental tool on the PC of a subject. Figure 1 illustrates the experimental tool's process. This tool records the subject's PC operations every $500 \mathrm{~ms}$. The tool presents a dialog window that interrupts the subject on the basis of interruption rules. The interrupted subject evaluates his or her interruptibility, which represents the extent to which the user can be interrupted at the timing, and inputs the evaluation into the dialog window.

The recorded operations are keystrokes, mouse clicks, mouse wheel usage, active window name (.exe name) process ID, window message (quit and clipboard), and the number of open windows. 
The tool's interruption rule is as follows: Interruptions are made at both AS and NAS. Interruptions at NAS are made when the subject has used one application continuously for more than $5 \mathrm{~min}$. Once the subject has been interrupted, the subject is not interrupted again for $10-15 \mathrm{~min}$.

When the subject is interrupted, he or she subjectively evaluates the interruptibility on a 5-point scale, from

"1: the user was absolutely uninterruptible" to " 5 : the user was absolutely interruptible." We instructed the subjects to imagine the interruption as a requirement for a 5-min conversation, and to evaluate the interruption timing without any reference to the frequency of the evaluation.

Sixteen office workers (including R\&D engineers, clerical staff, and managers) participated in the experiment in an office environment. We collected the operation records from each subject for at least $7 \mathrm{~h}$ per day. Each subject participated in the experiment for 14 days. We recorded more than $930 \mathrm{~h}$ of data in the office environment. We did not restrict the type of task that could be performed by the subject. Furthermore, we did not give subjects any instructions about the timing and frequency of AS, or the resumption of tasks. The subjects did their usual daily work such as entering data, programming, and writing reports or papers.

\section{Summary of collected data}

Table 1 summarizes the frequencies of each interruptibility score for AS and NAS, and their averages. Welch's t-test suggests that interruptions at AS are more acceptable than those occurring during NAS $[t(790)=1.72, p<0.1]$. The same tendency as in the previous experiments was confirmed [11]. However,

\begin{tabular}{|c|c|c|c|c|c|c|c|}
\hline & \multicolumn{3}{|c|}{ Subjective Interruptibility } & \multirow{2}{*}{ Total } & \begin{tabular}{c} 
Avg. \\
Interru \\
\cline { 2 - 6 }
\end{tabular} \\
\cline { 2 - 6 } & $\mathbf{2}$ Low & Mid & \multicolumn{2}{c|}{ High } & & ptibity \\
\hline AS & 104 & 64 & 80 & 100 & 111 & 459 & 3.1 \\
\hline NAS & 114 & 46 & 58 & 74 & 86 & 378 & 2.9 \\
\hline
\end{tabular}

Table 1. Subjective interruptibility scores during AS vs. NAS.

the interruptibility scores for AS varied widely from 1 to 5 , which indicates the differences between the ASs regarding cognitive cost.

\section{Analysis}

In this study, we approximated the transitions occurring between active applications as the switching that takes place between tasks.

\section{Resumption lag in previous studies}

Previous studies have used the RL as an index for cognitive cost caused by interruptions. RL is defined and calculated as the time between the timing of the task switch from the interrupted task to the other window and the onset of the operations related to the switched task [12]. In previous studies, the observed RLs were mainly 3-8 s. These experiments were performed with predetermined tasks within a limited duration. Subjects were instructed to perform a second task as soon after the task switching as possible. In other words, the experiments were designed to remove or control any factors that affect the RL, such as motivation. Thus, the RL is regarded as an index for the cognitive cost caused by interruptions. In contrast, we calculated RLs from the data collected in uncontrolled environments. Thus, the calculated RLs may be affected by factors other than the cognitive cost. 


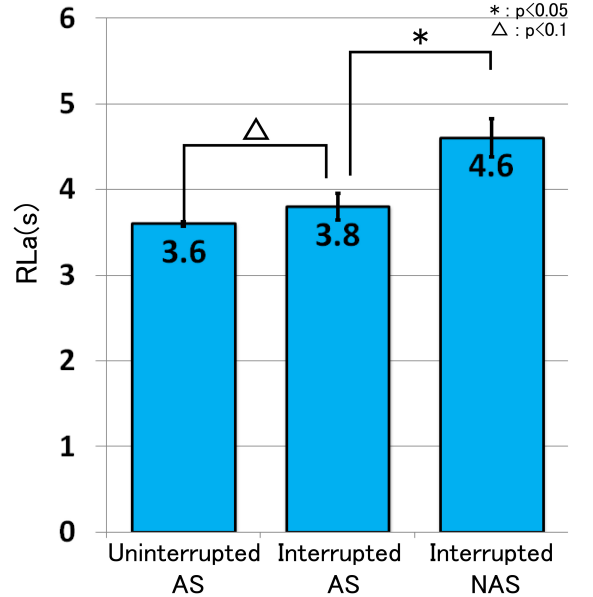

Figure 2. RLa of uninterrupted AS, interrupted AS, and interrupted NAS

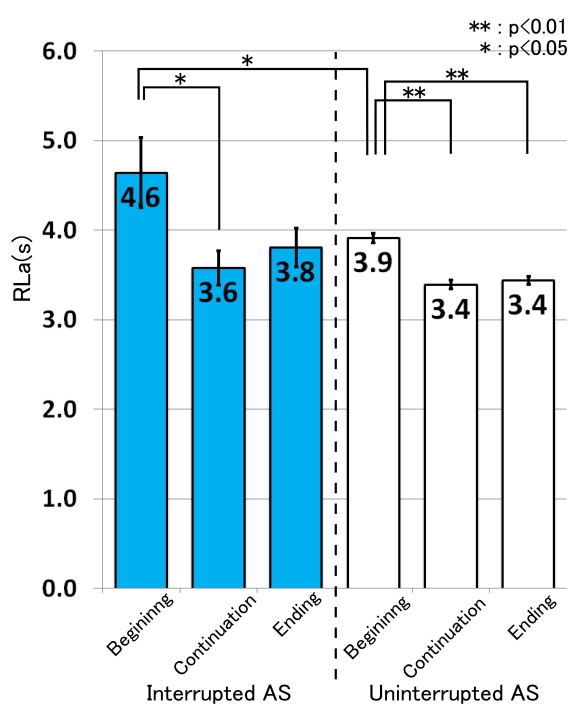

Figure 3. RLa of interrupted and uninterrupted AS at the beginning, continuation, and ending of task.
Definition of resumption lag in actual environment We defined the calculated RL for the uncontrolled environment as "the resumption lag in actual environment (RLa)," to distinguish from the RL in previous studies. We assumed that the working memory resumption begins at the task switches from the interruptibility evaluation dialog to another window. The end of the resumption process was defined as the observation of the first PC operation, which is similar to previous studies. To exclude the PC operation for task switching, the PC operations at the switching were ignored.

\section{$\mathrm{RLa}=\{$ time of first PC operation $\}$}

- \{time of task switching from evaluation dialog\}

\section{Results}

\section{Task phase and RL}

According to previous studies, the RLs for interruptions at AS are expected to be shorter compared with the RLs during NAS. Therefore, we compared the RLas for both AS and NAS.

Figure 2 shows the RLas at uninterrupted AS (no interruptions occurred at AS), interrupted AS, and interrupted NAS. The number of uninterrupted ASs was 13,475. The average RLa of uninterrupted AS was 3.6 $\mathrm{s}$; that of interrupted AS was $3.8 \mathrm{~s}$; and that of interrupted NAS was $4.6 \mathrm{~s}$. We applied the one-way analysis of variances and Bonferroni's multiple comparisons to the RLas. The results demonstrate that the RLa of an interrupted AS was longer than that of the uninterrupted AS $(p<0.1)$, and the RLa of the interrupted NAS was significantly longer than that of the interrupted AS $(p<0.01)$. Thus, we confirmed that the interruptions increase the cognitive cost, and the cognitive cost during AS is smaller than the cost during NAS in an uncontrolled environment.

As already shown in Table 1, in terms of cognitive cost, there are some variations to AS. In general, the amount of information transmitted is presumed to be small at task switching. The cognitive cost will decrease if less information is transmitted through the task switch. On the other hand, Jin and Dabbish [5] suggested that the AS, which is a self-interruption, occurs for several reasons. Iqbal and Bailey [4] revealed that task phase affects the cognitive cost. To examine the effects of task phases, we categorized the ASs into three groups, i.e., "beginning of task," "continuation of task," and "ending of task," on the basis of the increase or decrease in the number of opened windows after AS.

Figure 3 summarizes the RLa of the interrupted AS and uninterrupted AS for each task phase. The two-way analysis of variances and Bonferroni's multiple comparisons demonstrated that the interruption at the beginning of the task significantly increased the RLa

$(p<0.05)$. In contrast, the RLa at the ending and during the continuation of the task was not affected by interruptions. Therefore, we confirmed that the task phases affect the cognitive cost in an uncontrolled environment.

Interruptibility and $R L$

As mentioned in previous studies, the cognitive cost was expected to affect the subjective interruptibility during work. Thus, we analyzed the relationship between the interruptibility and RLa. Furthermore, to compare this assumption using another data set, we 


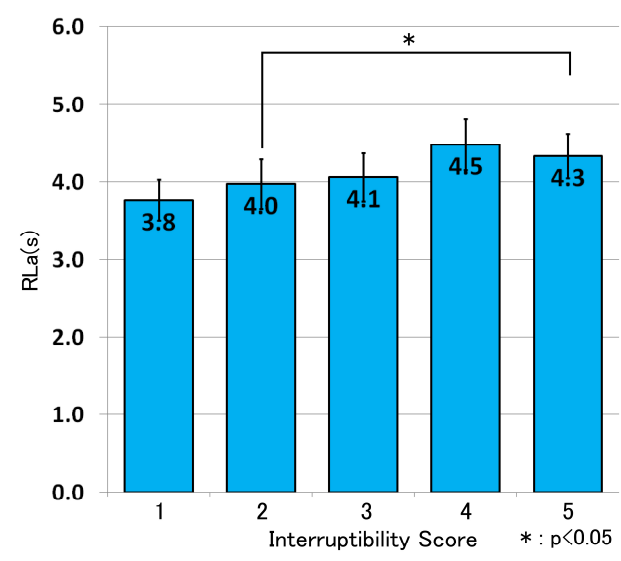

(a) Office Environment

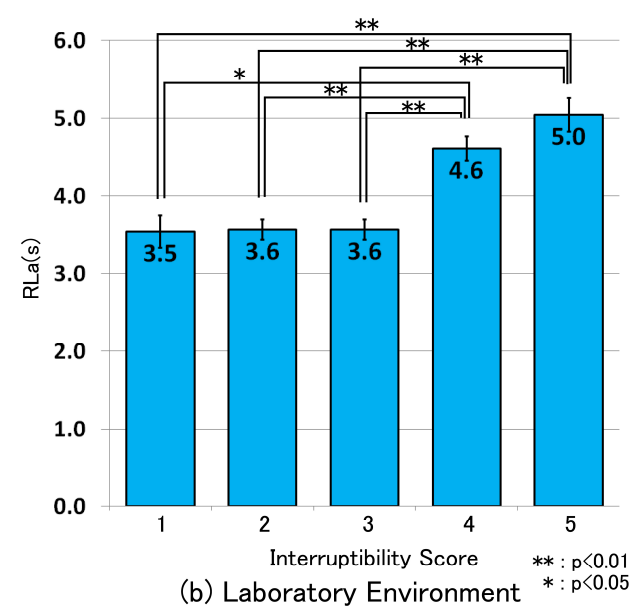

Figure 4. Relationship between interruptibility and RLa in (a) Office data set and (b) Laboratory data set collected another $50 \mathrm{~h}$ of data from 11 university students in a laboratory environment and analyzed the data set.

Figure 4 presents the results obtained by comparing the interruptibility score and RLa. In both data sets, the RLa at score 1 was shortest and the higher interruptible score also showed longer RLa. In the office data set, the average of RLa at score 1 was $3.8 \mathrm{~s}$. On the other hand, the average of RLa at score 4 was $4.5 \mathrm{~s}$, and that at score 5 was $4.3 \mathrm{~s}$. The RLa increased gradually according to the interruptibility. In the laboratory data set, the average of RLa at score 1 was $3.5 \mathrm{~s}$, which was almost within the same range as that of office workers at score 1 . However, the RLa at interruptible state, which includes score 4 and 5, were increased drastically compared with that of office workers.

We analyzed the RLa for both sets of data using oneway analysis of variances and Bonferroni's multiple comparisons. As shown in Figure 4, some significant differences were observed. The RLa length in the uninterruptible state was within the same range of the $\mathrm{RL}$ reported by previous studies $[8,10]$. However, the result suggested that RLa in interruptible state of the user tends to be longer than the RLa in uninterruptible state.

\section{Discussion}

The analysis results demonstrated that the

interruptions increased the RLa, and that the RLa of AS is significantly shorter than that of NAS. This suggested that the cognitive cost in uncontrolled environments is affected by interruptions which similar to that in previous studies. Furthermore, our analysis of the relationship between task phases and RLa reveals that interruptions at the end of a task hardly affected the RLa. As suggested in previous studies [4], at the end of the task, the amount of information transmitted after AS is presumed to be small. Thus, interruptions at the end of task do not increase the cognitive cost. From this result we infer that the task phase affects the cognitive cost in an uncontrolled environment.

According to the previous studies, the cognitive cost caused by interruptions decreases the worker's subjective interruptibility. In other words, a higher cognitive cost is expected to result in a longer $\mathrm{RL}$ and less interruptibility. However, the RLas for acceptable interruptions were longer, contrary to expectations in an uncontrolled environment. As seen in Figure 2, the increase of the cognitive cost caused by interruptions is obvious; thus, this suggests that subjective interruptibility in a realistic environment is more greatly affected by factors other than cognitive cost.

Mark et al., [7] reported that interruptions did not affect the time and quality of performed tasks, because subjects made an effort to recover the loss caused by interruptions. As one possible speculation from this result, the subject's attitude toward the work, such as motivation and concentration, decreases the RLa at low interruptible states. The length of the RLa at a low interruptible state is considered to be caused by realization of the potential of the subject who was highly motivated toward work. Otherwise, the subject made no attempt to resume the work immediately. In such states, interruptions are easily accepted. The differences in environments and in job awareness between students and employed workers may represent the difference in the RLa between the two data sets. 
At this time, other factors remain unpredictable; therefore, the worker's attitude is being speculated. These results suggest that, in addition to the cognitive cost, other user states or behaviors need to be recognized to accurately estimate interruptibility.

\section{Limitations}

The RLa was calculated in an uncontrolled environment. Thus, the RLa may be affected by factors other than the cognitive cost. The RLa is not strictly the same as $\mathrm{RL}$, and the interrupted task in this study was not memory-intensive, which may decrease the effective size. The analysis of other factors that affect the interruptibility is within the scope of future work.

\section{Conclusions}

In this study, we analyzed the RL in an actual environment. The results supported the expectation that task phase affects cognitive cost; the RL at AS was shorter than at NAS, and the RL at the beginning of the task is longer. However, interruptions at more acceptable ASs were longer, contrary to the expected result. This implied that factors other than the cognitive cost more strongly affected the interruptibility in a realistic environment.

\section{Acknowledgements}

This work was partly supported by KAKENHI, Japan Fund for Smart Space Technology toward Sustainable Society, and NICT commissioned research.

\section{References}

[1] Altman, E. M., and Trafton, J. G. Memory for goals: An activation-based model. Cognitive Science, 26(1)

(2002), 39-83.
[2] Cutrell, E. B., Czerwinski, M., and Horvitz, E. Effects of instant messaging interruptions on computing tasks. Proc. CHI2000 (2000), 99-100.

[3] Czerwinski, M., Horvitz, E., and Wilhite, S. A diary study of task switching and interruptions. Proc. $\mathrm{CHI}^{\prime} 04$ (2004), 175-182.

[4] Iqbal, S. T., and Bailey, B. P. Leveraging characteristics of task structure to predict costs of interruption. Proc. CHI2006 (2006), 741-750.

[5] Jin, J., and Dabbish, L. A. Self-interruption on the computer: A typology of discretionary task interleaving. Proc.CHI2009 (2009),1799-1808.

[6] Mark, G., Gonzalez, V. M., and Harris, J. No task left behind?: Examining the nature of fragmented work. Proc. CHI'05 (2005), 321-330.

[7] Mark, G., Gudith, D., and Klocke, U. The cost of interrupted work: More speed and stress. Proc.CHI2008 (2008), 107-110.

[8] Monk, C. A., Trafton, J. G., and Boehm-Davis, D. A. The effect of interruption duration and demand on resuming suspended goals. Journal of Experimenta Psychology: Applied, 14 (2008), 299-313.

[9] Salvucci, D. D., and Taatgen, N. A. Threaded cognition: An integrated theory of concurrent multitasking. Psychological Review, 115, 1 (2008), 101-130.

[10] Salvucci, D. D. On reconstruction of task context after interruption. Proc.CHI2010 (2010), 89-92.

[11] Tanaka, T., and Fujita, K. Study of user interruptibility estimation based on focused application switching, Proc. CSCW2011 (2011), 721-724.

[12] Trafton, J. G., Altman, E. M., Brock, P. and Mintz, F. E. Preparing to resume an interrupted task: Effects of prospective goal encoding and retrospective rehearsal, International Journal of Human-Computer Studies, 58 (2003), 583-603. 